\title{
Beyond MELD Predictors of Post TIPSS Acute Liver Failure the Lesson Learned
}

\author{
1 Department of Radio-Diagnosis, Max Super specialty Hospital and \\ Max Institute of Cancer Care, Vaishali, Ghaziabad, Uttar Pradesh, \\ India \\ ${ }^{2}$ Argim Group of Neurosciences, Artemis Hospital, Gurugram, \\ Haryana, India \\ ${ }^{3}$ Department of Radio-diagnosis, All India Institute of Medical \\ Sciences, New Delhi, India \\ Indian J Radiol Imaging 2021;31:618-622.
}

Ritu Verma ${ }^{1}$ Nishchint Jain ${ }^{2} \quad$ Abhishek Arora $^{3} \quad$ Shivanand Gamangatti $^{3} \quad$ Shailendra Chaturvedi ${ }^{1}$

\begin{abstract}
Address for correspondence Ritu Verma, MD, DNB, Department of Radio-Diagnosis, Max Super Specialty Hospital and Max Institute of Cancer Care Vaishali, Ghaziabad, Uttar Pradesh, India (e-mail: drritumamc@gmail.com).
\end{abstract}

\author{
Abstract

\section{Keywords} \\ - acute liver failure \\ - hepatic \\ encephalopathy \\ - PTLF \\ - TIPSS
}

TIPSS is safe and effective procedure for relieving portal hypertension by creating a low resistance portosystemic shunt. TIPSS reduces portal perfusion by 80 to $100 \%$ which then gradually gets partially compensated by increased flow from hepatic artery. Post TIPSS liver function shows brief deterioration which tends to start recovering in few weeks. However, progressive liver failure requiring emergency transplant or death remains a serious concern after TIPSS creation. The causes of post TIPSS liver failure are diverse and difficult to predict. Due to its rarity the definition of post TIPSS liver decompensation is also not well described in literature. Till date MELDNa score has been considered as the most reliable predictor of post TIPSS liver decompensation. In common practice post TIPSS liver failure is less likely in patients with model for endstage liver disease (MELD) score less than 18. We have experienced two unusual cases of post-TIPSS liver failure (PTLF) in patients with initial acceptable/low MELD score and the importance of non-MELD factors that may negatively influence post TIPSS outcome. Most of these can be routinely investigated prior to creating shunt thereby identifying patients at high risk of developing PTLF.

\section{Introduction}

Post TIPSS liver decompensation may adversely affect the clinical outcome and if severe it may also require TIPSS reduction, liver transplant or may lead to death. No definition for PTLF is currently available in clinical guidelines to identify these subsets of patients. Post procedure the enzymes show transient rise which tends to get stabilized within a few days. An exaggerated increase or delayed increase in enzyme levels may indicate irreversible liver cell injury or failure. Gradually the synthetic liver function also decreases due to diversion of blood flow from hepatocytes. According to International Study Group of Liver Surgery (ISGLS) post-hepatectomy liver failure is defined by an abnormal or increasing serum bilirubin and INR on or after postoperative day 5 , and further stratified into subgroups based on clinical outcomes. ${ }^{1}$ However, post TIPSS liver failure is yet to be properly defined in literature.

\section{Case 1}

A 26-year-old female presented with gradual abdominal distension of 1-year duration with history of multiple large
DOI https://doi.org/ 10.1055/s-0041-1736403. ISSN $0971-3026$.

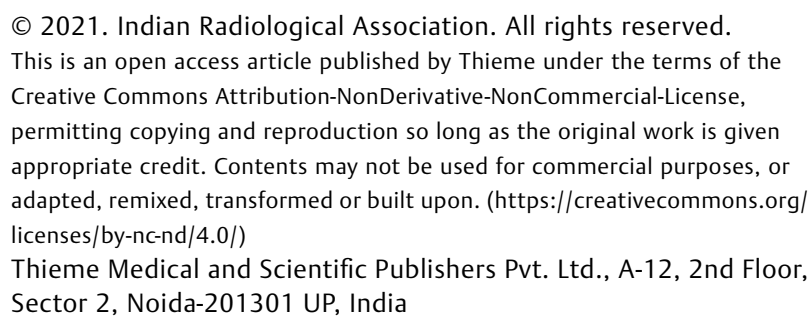




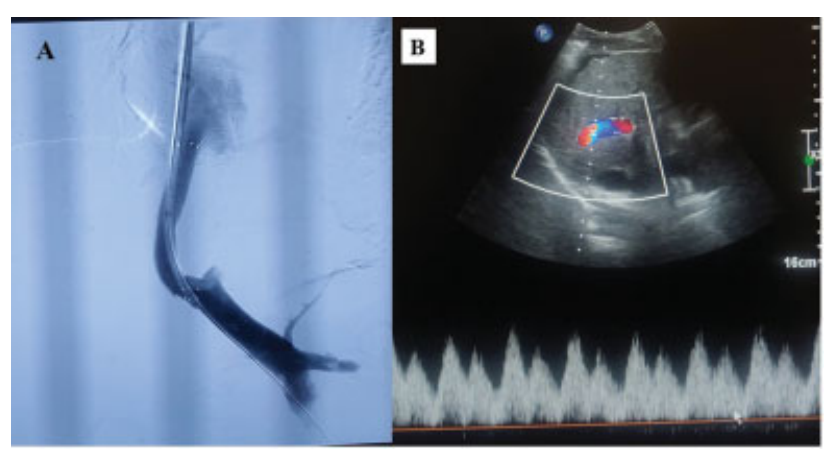

Fig. 1 Case 1: (A) DSA image post DIPSS creation in patient of cirrhosis due to diffuse BCS with no recanalizeable HV and a patent IVC and (B) post TIPSS USG Doppler showing typical phasic flow with velocity of $90 \mathrm{~cm} / \mathrm{s}$. IVC, inferior vena cava; USG, ultrasonography.

volume paracentesis for last 3 months. Viral markers and autoantibodies were negative. Ceruloplasmin and ferritin levels were normal. Doppler examination was done, which showed heteroechoic cirrhotic liver with diffuse obliteration of all three hepatic veins and normal IVC. Few small intrahepatic collaterals were seen. No recanalizable hepatic vein could be identified. There was gross ascites and gross right pleural effusion. The diagnosis of Budd Chiari syndrome with diffuse involvement of all three hepatic veins was made. Patient was worked up in hematology for hypercoagulable state which was negative. Due to recurrent tense ascites direct transjugular intrahepatic portosystemic shunt was planned. Routine investigations showed TLC 4400, $\mathrm{Hb}$ : $6.8 \mathrm{~g} \%$, platelet count $(65,000)$, bilirubin 1.1, INR: 2.1, albumin: $3.9 \mathrm{~g} \%$, urea: $32 \mathrm{mg} \%$, and creatinine $1.1 \mathrm{mg} \%$. Her pre-procedure MELDNa score was 11 and CPS was eight. Serum electrolytes and echocardiography were normal.

As hepatic vein stump was not identified, direct intrahepatic portosystemic shunt was done through inferior vena cava (-Fig. 1). The procedure was uneventful, and the patient was started on low molecular weight heparin. Day after, patient developed hypotension which later required inotropic support. There was no evidence of sepsis and USG did not reveal hemothorax, hemopericardium, or hemoperitoneum. Later she developed serial progressive increase in bilirubin from 1.1 to $3.5 \mathrm{mg} \%$, liver enzyme levels peaked to eight times above baseline with INR of 5.5. On day three, she developed hepatic encephalopathy which worsened from grade 1 to grade 4 within next 24 hours despite treatment. Work-up revealed normal electrolytes with no evidence of gastrointestinal bleed. Serum ammonia levels showed a gradual rising trend and later she was unable to maintain glucose levels and eventually died on day six, post TIPSS.

\section{Case 2}

A 55-year-old female with type II diabetes, chronic hepatitis C-related chronic liver disease requiring recurrent large volume paracentesis twice a week was referred for TIPSS. There was no history of upper gastrointestinal bleed and hepatic encephalopathy. USG Doppler revealed CLD with PHTN. All three hepatic veins and inferior vena cava (IVC) were normal. Her liver function tests showed total bilirubin of $1.9 \mathrm{mg} \%$ and serum albumin of $3.2 \mathrm{~g} \%$, serum sodium of $139(\mathrm{mEq} / \mathrm{L})$, and creatinine of $1.5 \mathrm{mg} \%$. There was mild anemia (Hb: $9.1 \mathrm{~g} \%$ ) and thrombocytopenia $(55,000 / \mathrm{mL})$. There was no history of cardiac illness. Her MELDNa score was nine and CPS was seven. TIPSS was performed as standard described technique with $10-\mathrm{mm}$ shunt diameter. Ascites disappeared in three following days; however, the patient developed grade 2 to $3 \mathrm{HE}$ which was managed medically. Patient recovered very gradually and was discharged after 10 days of medical management. She continued to have grade $1 \mathrm{HE}$ at home after discharge and after another week patient relapsed with grade two-thirds HE and was re-admitted. Her work-up revealed no sepsis, electrolyte imbalance and there was no evidence of GIB. She was treated medically with lactulose, bowel wash, rifampicin, and hepamerz. Despite all conservative efforts serum ammonia levels continue to rise and this time patient required prolonged hospitalization for around 2 weeks. Due to distress caused by unresponsive high grade HE, TIPSS reduction was planned. A 14-Fr long sheath was inserted into TIPSS via jugular route and two 6-mm SEMS were simultaneously deployed in the tract over two wires. One of the stents was closed with Amplatzer plug (-Fig. 2) leaving other stent patent, reducing the TIPS shunt diameter to 50\%. After shunt reduction, patient started developing ascites again and patient showed only some degrees of improvement in the status of hepatic encephalopathy. However, she continued to be in waxing and waning state of HE.

Careful history revealed past history of hypothyroidism, for which she took treatment for a couple of years and left around 2 years back. Her repeat thyroid profile revealed TSH of 46.4 and undetectable fT3 and fT4. She was initially started with $100 \mu \mathrm{g}$ intravenous thyroxine followed by oral thyroxine, after which she showed rapid improvement in next 48 hours. She was discharged in stable condition after 7 days and remained symptom free for next 2 months. Thereafter, she started requiring repeated paracentesis and eventually developed spontaneous bacterial peritonitis followed by progressive sepsis and multiorgan failure.

\section{Discussion}

Liver failure after TIPS insertion is a rare but fatal complication. Typical presentation is marked elevation in liver enzymes, coagulopathy, and severe hepatic encephalopathy. The risk of 3-month mortality after elective TIPS creation is $35 \%, 16 \%$, and virtually nil in patients with a MELDNa score of 18 , between 11 and 17 and with model for end-stage liver disease (MELD) score of 10 or less, ${ }^{2,3}$ respectively.

The actual etiology of hepatic decompensation after TIPS insertion is variable, but generally involves insult to an alreadycompromised liver in one or more of the following manners ${ }^{4}$ :

- TIPS insertion reduces or reverses portal perfusion resulting in some degree of hepatocyte ischemia.

- Possible compression or occlusion of hepatic artery by the metallic stent at porta resulting in ischemia or potential infarction. 


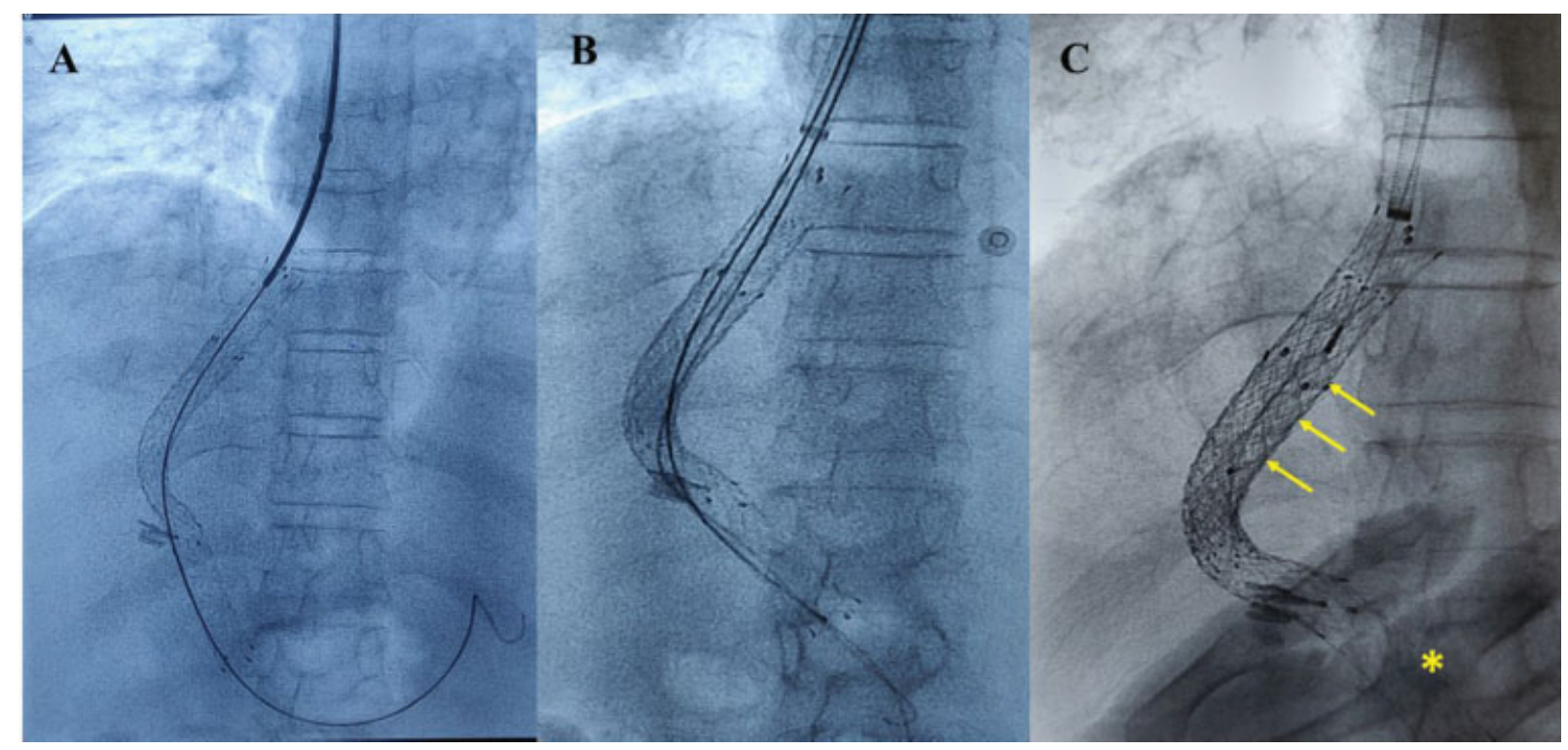

Fig. 2 CASE 2: (A) FluoroSpot image post TIPSS in HCV-related CLD with refractory ascites (note post cholecystectomy clips in situ). (B) Fluoro image showing two parallel wires after deployment of two separate smaller $6 \mathrm{~mm} \times 60 \mathrm{~mm}$ SEMS deployed within the pre-placed 10 -mm TIPSS stent. (C) Post shunt reduction image: Reduction done by occlusion of one of the stents with Amplatzer plug (arrow). Note hand artifact (Asterix).

- The covered portion of the TIPS can occlude one or more hepatic veins, particularly when there are shared origins, resulting in a HVOTO type of picture.

If no evidence of vessel occlusion or thrombosis is present on multiphase CT, the etiology may be most likely related to the altered portal venous flow dynamics, and thus urgent TIPS reduction/occlusion with coils or plugs should be attempted. Emergency liver transplant should be reserved for cases that deteriorate after conservative measures.

There are two limbs to prevent liver decompensation after a TIPSS creation. One is to identify high-risk patients prior to the procedure and the other is early identification of any treatable cause of impending PTLF. In patients with marginal hepatic functional status, one should be very careful in reducing the portosystemic gradient, since a large decrease may increase the risk of developing acute liver failure.

To identify patients at high risk of developing PTLF several prognostic scores are used in literature. Clinical and biochemical factors identified to be associated with poorer outcome including advanced age, history of encephalopathy, ascites, increased prothrombin time, elevated bilirubin level, low sodium and albumin levels, and emergent indication for TIPS rather than elective. Scoring systems like Child-Pugh score, MELD score, Emory score, and APACHE II scores have been used to help prognosticate and counsel patients being considered for TIPSS. In general, poorer post TIPSS outcome is expected with a Child-Pugh score $>12$, MELD score $>18$, Emory score $>3$, or an APACHE II score $>18$. High serum total bilirubin, high HVPG, and low albumin are independent factors for predicting mortality within 1 year ${ }^{5,6}$ according to various studies. MELD and MELDNa have performed best amongst all scores and form the criteria for patient selection in most of the centers. ${ }^{7}$ A score less than 18 is taken as ideal cut-off for performing a TIPSS procedure.

Development of PTLF in a patient with normal MELD score like in our cases requires careful consideration of other variables that may adversely affect the outcome as seen in our first patient. There is relative paucity of literature on the development of PTLF in patients having MELD score $<12 .{ }^{8}$ In a single center large retrospective study several other factors have been identified predicting poorer outcome even in patients with normal MELD score. They concluded that refractory ascites as indication versus GIB, low $\mathrm{Hb}$, and platelet count, MELD 11 or 12 versus less than or equal to ten, and CPS more than seven carry higher risk of developing PTLF. Moreover, there is no correlation between stent diameter and decrease in portosystemic pressure gradient. This was common to both cases having normal MELD score but severe anemia and thrombocytopenia that affected the outcome. In their study post TIPS early liver failure in patients with refractory ascites and a baseline MELD score of 11 or 12 varied from 14 to $60 \%$ according to change in the platelet count and from 14 to $69 \%$ according to change in the hemoglobin level. The effect of low platelet may reflect the severity of the hemodynamic abnormalities correlated with portal hypertension that are not immediately corrected by TIPS creation and the association of low hemoglobin and poor post TIPSS outcome is not well elaborated.

After appropriate pre-procedure patient selection, the second limb is early identification of any treatable cause of PTLF. The criteria for PTLF is another matter of debate. Different definitions have been proposed and a consensus criteria is yet to be universally accepted. AASLD defined ALF as "evidence of coagulation abnormality international normalized ratio for prothrombin time $>1.5$ and hepatic encephalopathy in a patient with a disease 
that has only been recognized for less than 26 weeks. ${ }^{9}$ Though ISGLS states the criteria for liver failure post resection, there is no consensus guidelines on the definition of what constitutes post TIPSS acute liver failure. Gaba and Lakhoo ${ }^{10}$ in their review of 268 patients formulated the definition of PTLF and concluded that PTLF was defined by $\geq$ threefold bilirubin and/or $\geq$ twofold INR elevation associated with clinical outcomes of prolonged hospitalization/increase in care level (grade 1), TIPS reduction or liver transplantation (grade 2), or death (grade 3 ) within 30-days of TIPS. The treatment remains primarily supportive and in extreme cases LTP is required.

After TIPSS rising ammonia causing HE as a part of liver decompensation is seen in as many as 30\% patients which remains a serious clinical concern. Usually, it responds to conservative management and only rarely it requires TIPSS reduction/closure or urgent liver transplant. Medically refractory HE first prompts complete clinical, biochemical, and metabolic work-up to rule out GIB, sepsis, and electrolyte imbalance which needs treatment. Our patient did not respond to all measures to reduce ammonia levels. This led to search for other causes of hyperammonemia, which revealed severe hypothyroidism as the possible cause. Till date there have been only few (eight) case reports on hypothyroidism causing hyperammonemic coma and all except one were patients of CLD. ${ }^{11}$ This further emphasizes that hypothyroidism precipitates hyperammonemia of liver dysfunction. However, none of them was post TIPSS creation though spontaneous portosystemic shunt has existed in one of these case reports. All of them have responded well to thyroxine replacement.

Inefficient urea synthesis during hypothyroidism is expected to result in an increased ammonia level. In liver failure and/or in patients with portal-systemic shunts, ammonia reaches the systemic circulation and the central nervous system. Ammonia is metabolized to glutamine in astrocytes through the glutamine synthetase enzyme and both ammonia and glutamine exert toxic effects on brain. ${ }^{12}$ An increase in liver enzymes concentration, AST more than ALT, is often found to be associated with hypothyroidism. Microscopic changes like central congestive fibrosis have also been described in myxedema states. ${ }^{13}$

Studies have also shown that the mean FT3 and FT4 levels are significantly decreased and the mean TSH levels were significantly increased in liver cirrhosis. Low levels of FT3 also correlate with the severity of liver disease in the form of CTP or MELD. TSH levels increase with increasing MELD score. Therefore, thyroid levels in cirrhotic patients may be used as a prognostic marker. It has been proposed that low FT3 and high TSH might be used as a predictor of mortality in liver cirrhosis. ${ }^{14}$

Though hypothyroidism rarely presents first as hyperammonemia but it may precipitate HE in existing CLD, more so after creating a TIPSS which further increases systemic ammonia levels as seen in our second case. Though there is no cut off value of TSH or fT4 levels which is acceptable for creating a TIPSS, thyroid function should still be evaluated and should be normalized before an elective TIPSS. Refracto-
Table 1 Patient profile pre-procedure

\begin{tabular}{|l|l|l|}
\hline & $\begin{array}{l}\text { Patient 1 baseline } \\
\text { profile }\end{array}$ & $\begin{array}{l}\text { Patient 2 baseline } \\
\text { profile }\end{array}$ \\
\hline $\begin{array}{l}\mathrm{CBC} \\
\text { (Hb/TLC/ } \\
\text { Platelet) }\end{array}$ & $6.8 / 4400 / 65,000$ & $9.1 / 3300 / 55000$ \\
\hline $\begin{array}{l}\text { LFT } \\
\text { (Bil/OT/PT/ } \\
\text { protein/alb) }\end{array}$ & $1.1 / 48 / 68 / 7.2 / 3 / 9$ & $1.9 / 186 / 180 / 7.1 / 3.2$ \\
\hline KFT (Ur/Cr) & $32 / 1 / 1$ & $111 / 1.5$ \\
\hline INR & 2.1 & 1.3 \\
\hline MELDNa & 11 & 9 \\
\hline CPS & 8 & 7 \\
\hline Others & - & TSH: 46.4 \\
\hline
\end{tabular}

ry HE work-up should include a complete thyroid profile and immediate correction if hypothyroidism is detected.

These discussed non-MELD factors, like severe anemia, thrombocytopenia, and hypothyroidism, are important to be evaluated prior to the procedure so as to prevent liver decompensation and reduce early post TIPSS mortality.

\section{Conclusion}

In our cases despite a favorable low MELD score ( - Table $\mathbf{1}$ ) both patients developed progressive liver failure and death implicating other causes which are beyond consideration by MELD alone. Despite abundance of prognostic scores available for post TIPSS survival in CLD patients, careful patient selection is crucial for clinical success. In patients with normal MELD score low $\mathrm{Hb}$, platelet and resistant ascites as indication may pose additional risk for PTLF. Thyroid function should be evaluated prior to shunt creation and euthyroid status should be ensured prior to elective TIPSS. In cases of refractory hyperammonemia complete thyroid profile is required in addition to the standard work-up. Unlike the traditional concept of refusing TIPSS in patients with MELD score above 18 according to newer concept these patients may be taken for TIPSS after careful patient selection and detailed discussion with the family. The positive outcome and decrease in incremental risk of death ultimately benefit this subgroup of patients as well as demonstrated in some of the newer studies. ${ }^{15}$

\section{Financial Support and Sponsorship}

Nil.

Conflict of Interest

None declared.

\section{Acknowledgments}

The authors thank all the residents and physicians who contributed to the study of the reported case and to the editors of IJRI for giving the opportunity to publish this case report. 


\section{References}

1 Rahbari NN, Garden OJ, Padbury R, et al. Posthepatectomy liver failure: a definition and grading by the International Study Group of Liver Surgery (ISGLS). Surgery 2011;149(05):713-724

2 Ferral H, Gamboa P, Postoak DW, et al. Survival after elective transjugular intrahepatic portosystemic shunt creation: prediction with model for end-stage liver disease score. Radiology 2004; 231(01):231-236

3 Lodato F, Berzigotti A, Lisotti A, et al. Transjugular intrahepatic portosystemic shunt placement for refractory ascites: a singlecentre experience. Scand J Gastroenterol 2012;47(12): 1494-1500

4 Salerno F, Merli M, Cazzaniga M, et al. MELD score is better than Child-Pugh score in predicting 3-month survival of patients undergoing transjugular intrahepatic portosystemic shunt. J Hepatol 2002;36(04):494-500

5 Dhanasekaran R, Gonzales P, West J, et al. Predictors of early mortality post transjugular intrahepatic portosystemic shunts and the role of hepatic venous pressure gradient. Int J Gastrointest Interv 2012;1:63-68

6 Ascha M, Abuqayyas S, Hanouneh I, et al. Predictors of mortality after transjugular portosystemic shunt. World J Hepatol 2016;8 (11):520-529

7 Allegretti AS, Frenk NE, Li DK, et al. Evaluation of model performance to predict survival after transjugular intrahepatic portosystemic shunt placement. PLoS One 2019;14(05):e0217442
8 Luca A, Miraglia R, Maruzzelli L, D’Amico M, Tuzzolino F. Early liver failure after transjugular intrahepatic portosystemic shunt in patients with cirrhosis with model for end-stage liver disease score of 12 or less: Incidence, outcome, and prognostic factors. Radiology 2016;280(02):622-629

9 Saliba F, Samuel D. Acute liver failure: current trends. J Hepatol 2013;59(01):6-8

10 Gaba RC, Lakhoo J. What constitutes liver failure after transjugular intrahepatic portosystemic shunt creation? A proposed definition and grading system. Ann Hepatol 2016;15(02):230-235

11 Díaz-Fontenla F, Castillo-Pradillo M, Díaz-Gómez A, et al. Refractory hepatic encephalopathy in a patient with hypothyroidism: another element in ammonia metabolism. World J Gastroenterol 2017;23(28):5246-5252

12 Thobe N, Pilger P, Jones MP. Primary hypothyroidism masquerading as hepatic encephalopathy: case report and review of the literature. Postgrad Med J 2000;76(897):424-426

13 Baker A, Kaplan M, Wolfe H. Central congestive fibrosis of the liver in myxedema ascites. Ann Intern Med 1972;77(06):927-929

14 Punekar P, Sharma AK, Jain A. A study of thyroid dysfunction in cirrhosis of liver and correlation with severity of liver disease. Indian J Endocrinol Metab 2018;22(05):645-650

15 Spengler EK, Hunsicker LG, Zarei S, Zimmerman MB, Voigt MD. Transjugular intrahepatic portosystemic shunt does not independently increase risk of death in high model for end stage liver disease patients. Hepatol Commun 2017;1(05):460-468 